\title{
DCA-Based Real-Time Residual Useful Life Prediction for Critical Faulty Component
}

\author{
Funa Zhou, Jiayu Wang, and Yulin Gao \\ School of Computer and Information Engineering, Henan University, Kaifeng, China \\ Correspondence should be addressed to Jiayu Wang; wangjiayu11115@163.com and Yulin Gao; gaoyulinhn@163.com
}

Received 28 February 2017; Accepted 26 April 2017; Published 21 May 2017

Academic Editor: Chaojie Li

Copyright ( 2017 Funa Zhou et al. This is an open access article distributed under the Creative Commons Attribution License, which permits unrestricted use, distribution, and reproduction in any medium, provided the original work is properly cited.

\begin{abstract}
Residual useful life (RUL) prediction is significant for condition-based maintenance. Traditional data-driven RUL prediction method can only predict fault trend of the system rather than RUL of a specific system component. Thus it cannot tell the operator which component should be maintained. The innovation of this paper is as follows: (1) Wavelet filtering based method is developed for early detection of slowly varying fault. (2) Designated component analysis is introduced as a feature extraction tool to define the fault precursor of a specific component. (3) Exponential life prediction model is established by nonlinear fitting of the historical RUL and the fault size characterized by the statistics used. Once online detection statistics is obtained, real-time RUL of the critical component can be predicted online. Simulation shows the effectiveness of this algorithm.
\end{abstract}

\section{Introduction}

With the rapid development of modern industrial technology, reliability, maintainability, and security of largescale system have widely received attention [1-4]. Abnormal detection, root cause identification, and RUL prediction are the stages for efficient system monitoring.

Abnormality is expected to be detected as quickly as possible to prevent major accidents and reduce loss of downtime and maintenance. In the past decades, multivariate statistical analysis techniques such as principal component analysis (PCA) and partial least squares (PLS) [5-7] have been widely used in complex system monitoring [8]. However, abnormal detection and fault diagnosis usually answer the question of "having trouble," not "how long it will happen."

Detection and maintenance after system failure are usually too late for system with higher security requirement and expensive downtime loss, such as power station, power transmission, petrochemical industry, large-scale iron, steel enterprise, and other industry fields [9].

If condition-based maintenance rather than breakdown maintenance is used, severe faults leading to shutdown can be avoided, and a lot of maintenance cost can be saved [10]. Fault prediction technology is a critical step of condition-based maintenance. In recent years, fault prediction has become a hot topic in the field of system monitoring [11]. Artificial neural network (ANN) [12-17], autoregressive model (AR) $[18,19]$, support vector machine (SVM) [20], vector autoregressive model (VAR), and so forth [21] are commonly used fault prediction methods. But these pure data-driven methods without reference to expert experience can only predict fault trend rather than directly predict RUL online.

An important stage of RUL prediction strategy is how to properly assess fault size that reflects the degradation process. If a damage precursor is available, damage precursor based method for RUL prediction is an objective choice [22]. So fault damage precursor as well as the control limits should be determined before online RUL prediction. Existing method for establishing the fault damage precursor can be categorized into 2 classes: data-driven method and model-based method. Accurate fault propagation model to establish fault damage precursor is usually unavailable. However, thanks to the rapid development of sensor technology and condition monitoring technology, large amount of observation data reflecting status of the system can be used to establish damage precursor by using data-driven method $[9,13]$. Therefore, data-based method is gradually attracting the attention of researchers. $\mathrm{Li}$ et al. use historical normal data to establish PCA model, and 1norm of the residual vector is used to define the fault damage precursor $[23,24]$. RUL can be estimated by multistep 
recursive prediction using AR model with forgetting factor $[25,26]$. Ma et al. [27] use square prediction error based fault reconstruction method to estimate fault size in the first step. Then RUL can be estimated by multilevel recursive prediction. Li et al. [24] propose a fault prediction method for industry process with performance degradation by using multiscale PCA. Fault size computed by fault reconstruction can be used to establish a prediction model based on exponential smoothing technique. These methods share the same deficiency that fault direction is computed by PCA related method [27]. But pattern compounding problem of PCA makes it impossible to establish 1-1 correspondence between the failure component and the reconstructed fault direction [28]. Thus PCA based RUL prediction cannot tell the operator which critical component should be maintained.

Designated component analysis (DCA) is a knowledgeguided data-driven feature extraction method which can be used to diagnose root cause of the abnormality [29]. In this paper, DCA is introduced as a feature extraction tool for establishing fault damage precursor and life prediction model. Since small fault is usually buried in noise, pretreatment of filtering is developed for early detection of slowly varying small fault.

Remark 1. It is assumed that failure of critical component defined by expert has disastrous impact on the system. So RUL prediction of critical component is much significant in the sense that unnecessary surplus maintenance can be reduced to save maintenance cost.

The remainder of this paper is organized as follows: Section 2 describes principal component analysis and designated component analysis. DCA-based real-time prediction for critical component is developed in Section 3. Simulation analysis in Section 4 shows the efficiency of the method proposed. Conclusions and further research are given in the last section of the paper.

\section{Review of PCA and DCA}

2.1. PCA Modeling. Assume $Y_{0} \in R^{n \times m}$ is the observation data matrix collected in the normal operation conditions, where $m$ is the number of observation variables and $n$ is the number of samples. Firstly, $Y_{0}$ is standardized via

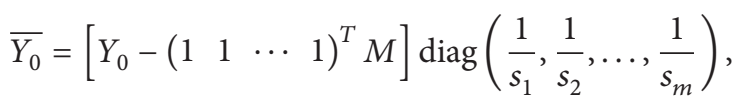

where $M=\left[Y_{0}(1) Y_{0}(2) \cdots Y_{0}(m)\right]$ is the mean of $Y_{0}, Y_{0}(m)$ is the variable of $Y_{0}, s=\left[\begin{array}{llll}s_{1} & s_{2} & \cdots & s_{m}\end{array}\right]$ is the standard deviation of $Y_{0}$.

$\overline{Y_{0}}$ can be decomposed into two parts as follows [11]:

$$
\overline{Y_{0}}=\sum_{i=1}^{v} t_{i} p_{i}^{T}+E,
$$

where $t_{i} \in R^{n \times 1}$ is the score vector, $p_{i} \in R^{m \times 1}$ is the loading vector, $v(v<m)$ is the number of significant principal components, and $E=\sum_{i=v+1}^{m} t_{i} p_{i}{ }^{T}$ is the residual error.
After establishing PCA modeling under normal operating conditions, multivariate statistics called squared prediction error (SPE), can be used for fault detection and diagnosis. SPE is described as follows:

$$
\operatorname{SPE}(i)=\|E(i)\|^{2}=Y_{i}\left(I-P P^{T}\right) Y_{i}^{T}
$$

where $Y_{i}(i=1,2, \ldots, n)$ represents a sample of online observation data, and $P$ is a matrix composed of the first $v$ loading vectors.

SPE control limit can be determined via

$$
\begin{aligned}
& Q_{\alpha}=\theta_{1}\left[\frac{C_{\alpha} \sqrt{2 \theta_{2} h_{0}^{2}}}{\theta_{1}}+1+\frac{\theta_{2} h_{0}\left(h_{0}-1\right)}{\theta_{1}^{2}}\right]^{1 / h_{0}}, \\
& \theta_{t}=\sum_{j=k+1}^{m} \lambda_{j}^{t} \quad(t=1,2,3), \\
& h_{0}=1-\frac{2 \theta_{1} \theta_{3}}{3 \theta_{2}^{2}}
\end{aligned}
$$

where $\lambda_{j}$ is the eigenvalues of the covariance matrices and $C_{\alpha}$ is the threshold of hypothesis testing to normal distribution with confidence level $\alpha$.

The system is considered to be abnormal if

$$
\mathrm{SPE} \geq Q_{\alpha}
$$

2.2. DCA. DCA is a knowledge-guided multivariate statistical feature extraction method [29]. The basic idea is to define a designated pattern via the fault-symptom relation.

First, different from the loading vector $p_{i} \in R^{m \times 1}$ of PCA, the definition of designated pattern $D_{i}$ is defined by a knowledge-guided means via

$$
D_{i}=\left[\begin{array}{c}
d_{i 1} \\
d_{i 2} \\
\vdots \\
d_{i m}
\end{array}\right], \quad i=1,2, \ldots, L
$$

where $d_{i r}(r=1,2, \ldots, m)$ can be taken as 0 or 1 according to the fault-symptom relation, and $d_{i r}=1$ means that the symptom $r$ is shown in the $i$ th designated pattern; otherwise $d_{i r}=0$.

Table 1 lists the typical fault-symptom relation of an air compressor. There are 17 typical faults and 19 observation parameters included in the table. Insufficient supply of lubricating oil or oil blockage, lubricant contamination, motor drive failure, and so on are the typical faults listed in Table 1. First-stage exhaust temperature, intercooler exhaust temperature, two-stage exhaust temperature, oil cooling outlet temperature and motor current, and so forth are the observed parameters [29]. 
TABLE 1: Typical fault-symptom relation of air compressor.

\begin{tabular}{ccccccccccccccccccccc}
\hline & $u_{1}$ & $u_{2}$ & $u_{3}$ & $u_{4}$ & $u_{5}$ & $u_{6}$ & $u_{7}$ & $u_{8}$ & $u_{9}$ & $u_{10}$ & $u_{11}$ & $u_{12}$ & $u_{13}$ & $u_{14}$ & $u_{15}$ & $u_{16}$ & $u_{17}$ & $u_{18}$ & $u_{19}$ & $u_{20}$ \\
\hline$D_{1}$ & 1 & 0 & 0 & 0 & 0 & 0 & 1 & 0 & 0 & 0 & 0 & 0 & 0 & 0 & 0 & 0 & 0 & 0 & 0 & 0 \\
$D_{2}$ & 0 & 1 & 0 & 0 & 0 & 0 & 1 & 0 & 0 & 0 & 0 & 0 & 0 & 0 & 0 & 0 & 0 & 0 & 0 & 0 \\
$D_{3}$ & 0 & 0 & 1 & 0 & 0 & 0 & 1 & 0 & 0 & 0 & 0 & 0 & 0 & 0 & 0 & 0 & 0 & 0 & 0 & 0 \\
$D_{4}$ & 0 & 0 & 0 & 0 & 0 & 0 & 0 & 0 & 0 & 0 & 1 & 0 & 1 & 0 & 0 & 0 & 0 & 0 & 0 & 0 \\
$D_{5}$ & 0 & 0 & 0 & 0 & 0 & 0 & 1 & 0 & 0 & 0 & 0 & 0 & 0 & 1 & 0 & 0 & 0 & 0 & 0 & 0 \\
$D_{6}$ & 1 & 1 & 1 & 0 & 0 & 0 & 1 & 0 & 0 & 0 & 0 & 0 & 0 & 0 & 0 & 0 & 0 & 0 & 0 & 0 \\
$D_{7}$ & 0 & 0 & 0 & 1 & 1 & 1 & 0 & 1 & 1 & 1 & 0 & 0 & 0 & 0 & 0 & 0 & 0 & 0 & 0 & 0 \\
$D_{8}$ & 0 & 0 & 0 & 0 & 1 & 1 & 0 & 0 & 0 & 1 & 0 & 0 & 1 & 0 & 0 & 0 & 0 & 0 & 0 & 0 \\
$D_{9}$ & 0 & 0 & 0 & 1 & 1 & 1 & 0 & 1 & 1 & 1 & 1 & 1 & 0 & 0 & 0 & 0 & 0 & 0 & 0 & 0 \\
$D_{10}$ & 0 & 0 & 0 & 0 & 0 & 1 & 0 & 0 & 0 & 1 & 0 & 0 & 1 & 0 & 0 & 0 & 0 & 0 & 0 & 0 \\
$D_{11}$ & 0 & 0 & 0 & 0 & 0 & 0 & 0 & 0 & 0 & 0 & 1 & 1 & 0 & 0 & 0 & 1 & 0 & 0 & 0 & 0 \\
$D_{12}$ & 0 & 0 & 0 & 0 & 0 & 0 & 0 & 0 & 0 & 0 & 0 & 0 & 1 & 0 & 0 & 0 & 0 & 0 & 0 & 0 \\
$D_{13}$ & 0 & 0 & 0 & 0 & 0 & 0 & 0 & 0 & 0 & 0 & 0 & 0 & 0 & 0 & 0 & 1 & 1 & 0 & 0 & 0 \\
$D_{14}$ & 0 & 0 & 0 & 0 & 0 & 0 & 0 & 0 & 0 & 0 & 0 & 0 & 0 & 0 & 0 & 0 & 1 & 0 & 0 & 0 \\
$D_{15}$ & 0 & 0 & 0 & 0 & 0 & 0 & 0 & 0 & 0 & 0 & 0 & 0 & 0 & 0 & 0 & 0 & 0 & 1 & 0 & 0 \\
$D_{16}$ & 0 & 0 & 0 & 0 & 0 & 0 & 0 & 0 & 0 & 0 & 0 & 0 & 0 & 0 & 0 & 0 & 0 & 0 & 1 & 0 \\
$D_{17}$ & 0 & 0 & 0 & 0 & 0 & 0 & 0 & 0 & 0 & 0 & 0 & 0 & 0 & 0 & 0 & 0 & 0 & 0 & 0 & 1 \\
\hline
\end{tabular}

From Table 1, the fifth row corresponds to the fault of insufficient supply of lubricating oil, the sixth row corresponds to the fault of lubricant contamination, and these fault patterns can be defined as follows [29]:

$D_{5}$

$=\left[\begin{array}{llllllllllllllllllll}0 & 0 & 0 & 0 & 0 & 0 & 1 & 0 & 0 & 0 & 0 & 0 & 0 & 1 & 0 & 0 & 0 & 0 & 0 & 0\end{array}\right]^{T}$,

$D_{6}$

$=\left[\begin{array}{llllllllllllllllllll}1 & 1 & 1 & 0 & 0 & 0 & 1 & 0 & 0 & 0 & 0 & 0 & 0 & 1 & 0 & 0 & 0 & 0 & 0 & 0\end{array}\right]^{T}$.

Then, project the observation variable $y$ to designated patterns $D_{i}$ to obtain the designated component $w_{i}$ :

$$
w_{i}=D_{i}^{T} y .
$$

Similarly to (2), the observation matrix $Y_{0}$ can be decomposed as

$$
Y_{0}=\sum_{i=1}^{L} D_{i} W_{i}+E
$$

where $L$ is the number of designated patterns, $W_{i}=$ $\left[w_{i}(1), w_{i}(2), \ldots, w_{i}(n)\right] \in R^{1 \times n}$, and $E$ is the residual matrix.

Finally, Shewhart chart of each designated component is used to implement fault diagnosis.

\section{DCA-Based Real-Time Prediction for Critical Component}

DCA-based RUL prediction method needs to determine critical component by expert experience. In this paper, the component corresponding to designated pattern $D_{1}$ is assumed to be a critical component.

\subsection{Fault Damage Precursor Based on Historical Observation}

3.1.1. Knowledge-Guided Data-Driven Feature Extraction. Project historical normal observation data $Y_{0}$ to the designated pattern $D_{i}$ to obtain the designated component vector $W_{0 i}$ :

$$
W_{0 i}=D_{i}^{T} Y_{0}, \quad i=1,2, \ldots, L .
$$

The designated component $W_{0 i}$ is the feature extracted from $Y_{0}$. In general, statistical distribution of $W_{0 i}$ is normal distribution as long as the observation of each observation variable is normal distribution.

3.1.2. Data-Driven Fault Damage Precursor. In the case when there is no fault damage precursor determined by expert or by physical fault propagation model, it is necessary to establish a data-driven fault damage precursor for describing the fault evolution process. For this purpose, the failure control limit $q$ to determine the failure time and the fault trend control limit $l_{1}$ for early detection of slowly varying fault should first be determined. Specific steps are as follows.

Step 1 (failure control limit and fault trend control limit). As it is analyzed in Section 3.1.1, $W_{0 i}$ has the normal distribution. By $3 \sigma$ criteria of hypothesis testing to normal distribution, the critical designated component $W_{01}$ falls into interval $[-3 \sigma, 3 \sigma]$ with confidence level $99.7 \%[30,31]$ :

$$
P\left(-3 \sigma \leq W_{01} \leq 3 \sigma\right)=99.7 \% \text {. }
$$

So the failure control limit $q$ can be determined via

$$
q=3 \sigma,
$$

where $\sigma$ is the standard deviation of $W_{01}$. 
For the sake of determining fault trend control limit $l_{1}$, wavelet filtering is firstly introduced as a preprocessing tool to historical normal observation data, and then DCA is used to perform feature extraction to the filtered normal observation. The filtering process can be described as follows $[32,33]$.

First, discrete wavelet transform (DWT) for the observation vector of the $i$ th observation variable $Y_{0}^{(i)}$ can be depicted via

$$
\begin{aligned}
& a_{(j)}(k)=\sum_{N} h(N-2 k) Y_{0_{(j)}}^{(i)}(k), \\
& d_{(j)}(k)=\sum_{N} g(N-2 k) Y_{0_{(j)}}^{(i)}(k),
\end{aligned}
$$

where $k=0,1,2, \ldots, n-1 ; n$ is the number of discrete sampling points; $a_{(j)}(k)$ is the scaling coefficient for signal on the scale $j ; d_{(j)}(k)$ is the wavelet coefficient for signal on the scale $j ; h$ is a low-pass filter, $g$ is a high-pass filter, and $N$ is the length of the filter.

After DWT, the next step is filtering. How to select the threshold is the key problem because it will directly affect the result of wavelet filter. In this paper, we choose the soft threshold method shown in

$$
\begin{aligned}
& \widehat{d}_{(j)}(k) \\
& \quad= \begin{cases}\operatorname{sign}\left(d_{(j)}(k)\right)\left(\left|d_{(j)}(k)\right|-T\right), & \left|d_{(j)}(k)\right| \geq T \\
0, & \left|d_{(j)}(k)\right|<T,\end{cases}
\end{aligned}
$$

where $T$ is the filtering threshold.

Finally, inverse discrete wavelet transform (IDWT) is implemented to obtain the filtered observation:

$$
\tilde{Y}_{0}^{(i)}=\sum_{k} a_{j}(k) h(N-2 k)+\sum_{k} \widehat{d}_{j}(k) g(N-2 k) .
$$

Based on the filtered normal observation, fault trend control limit $l_{1}$ can be determined via (19):

$$
\begin{aligned}
W_{0 i F} & =D_{i}^{T} \tilde{Y}_{0}, \quad i=1,2, \ldots, L, \\
l_{1} & =3 \sigma_{1},
\end{aligned}
$$

where $\sigma_{1}$ is the standard deviation of $W_{01 F}$.

Step 2 (fault trend detection point and the failure point). Project historical faulty observation data $Y_{1}$ to the designated pattern $D_{1}$ to get the designated component $W_{1}$ :

$$
W_{1}=D_{1}^{T} Y_{1}
$$

The extracted feature $W_{1}$ can characterize the fault evolution process of the critical component. The failure point $t_{f}$ can be calculated as follows:

$$
t_{f}=\min \left\{k: W_{1}(k) \geq q\right\} .
$$

Similarly, the filtered designated component can be computed via (22).

$$
W_{1 F}=D_{1}^{T} Y_{1 F}
$$

Once $W_{1 F}$ and fault trend control limit $l_{1}$ have been obtained, the definition of fault trend detection point $t_{s}$ can be expressed as

$$
t_{s}=\underset{k}{\operatorname{argmin}}\left\{k: W_{1 F}(k) \geq l_{1}\right\} .
$$

Step 3 (fault damage precursor). Smoothing technique descripted in (24) is required to get a more smooth fault damage precursor:

$$
\begin{aligned}
& \overline{W_{1 F}}(1)=W_{1 F}(1) \\
& \overline{W_{1 F}}(2)=\frac{\left(W_{1 F}(1)+W_{1 F}(2)+W_{1 F}(3)\right)}{3} \\
& \overline{W_{1 F}}(3) \\
& =\frac{\left(W_{1 F}(1)+W_{1 F}(2)+W_{1 F}(3)+W_{1 F}(4)+W_{1 F}(5)\right)}{5} \\
& \vdots \\
& \overline{W_{1 F}}(k) \\
& =\frac{\left(W_{1 F}(k-c+1)+W_{1 F}(k-c+2) \cdots+W_{1 F}(k)\right)}{c} \\
& \vdots,
\end{aligned}
$$

where $c$ is the size of the smoothing window; $W_{1 F}(i)(i=$ $1,2, \ldots, n)$ is the element of vector $W_{1 F}$.

The fault evolution process in the period from $t_{s}$ to $t_{f}$ can be defined as fault damage precursor, denoted by $C$ :

$$
C\left(t-t_{s}+1\right)=\overline{W_{1 F}}(t), \quad\left(t=t_{s}, t_{s}+1, \ldots, t_{f}\right) \text {. }
$$

3.1.3. The RUL Prediction Model. The historical RUL is defined by the deviation between $t$ and $t_{f}$ :

$$
\operatorname{RUL}\left(t-t_{s}+1\right)=t_{f}-t, \quad\left(t=t_{s}, t_{s}+1, \ldots, t_{f}\right),
$$

where $t$ is the current sampling time.

Once the fault precursor defined in (26) is established, the algorithm of establishing RUL prediction model can be divided into three steps.

Step 1. Computerize the deviation between fault damage precursor and the control limit denoted by $R$ :

$$
R\left(t-t_{s}+1\right)=C(t)-l_{1}
$$

Step 2. Once the deviation $R$ and the historical RUL have been obtained, historical data can be determined as follows:

$$
J=\{(R, \mathrm{RUL})\} .
$$

Step 3. Exponential life prediction model is established by nonlinear fitting of the historical data. Fitting equation is shown as follows:

$$
\mathrm{RUL}=c+a \times e^{b R} .
$$


3.2. Online Fault Diagnosis. Firstly, online observation data after pretreatment of wavelet filtering is denoted by $Y_{z}$. Project $Y_{z}$ to the designated patterns $D_{i}$ to obtain the designated component vector $W_{i z}$ :

$$
W_{i z}=D_{i}^{T} Y_{z}, \quad i=1,2, \ldots, L .
$$

The smoothed designated component is denoted by $\overline{W_{i z}}$. Shewhart charts based method is used in this paper to implement fault diagnosis.

3.3. Online Life Prediction. A key step of online RUL prediction for the critical component is to judge whether $R(k)$ is positive or negative. If $R(k)$ is positive, it means that the system is abnormal. Real-time RUL prediction results can be obtained via exponential life prediction model:

$$
\begin{aligned}
R(k) & =\overline{W_{1 z}}(k)-l_{1}>0, \\
\widehat{\operatorname{RUL}}(k) & =c+a \times e^{b R(k)}, \quad \\
& k=t_{s}, t_{s}+1, \ldots, t_{f} .
\end{aligned}
$$

\section{Simulation}

4.1. Simulation Data. In this section, $m=20, n=1000$ are used for simulation. Normal observation data is the compound of ten coexisting change patterns:

$$
Y_{0}=\sum_{i=1}^{L} D_{i} \overline{W_{i}}
$$

where $L=10$ is the number of designated patterns, $D_{1}, D_{7}$ represent fault patterns, and the rest are normal random disturbance patterns. $\bar{W}_{i}$ is the sample vector of designated component for simulation. In MATLAB, $\bar{W}_{i}$ can be defined by the function of "randn" and some linear operations. Assuming $\overline{W_{i}}$ is normally distributed,

$$
\begin{aligned}
& \overline{W_{1}}=\operatorname{randn}(1, n), \\
& \overline{W_{2}}=0.5 \overline{W_{1}}+0.8 \operatorname{randn}(1, n), \\
& \overline{W_{3}}=0.5 \overline{W_{1}}+0.5 \overline{W_{2}}, \\
& \overline{W_{4}}=0.5 \overline{W_{2}}+0.1 \operatorname{randn}(1, n), \\
& \overline{W_{5}}=\overline{W_{3}}+0.2 \overline{W_{4}}, \\
& \overline{W_{6}}=0.2 \overline{W_{1}}+0.3 \overline{W_{4}}, \\
& \overline{W_{7}}=0.3 \operatorname{randn}(1, n), \\
& \overline{W_{8}}=0.2 \overline{W_{7}}+0.2 \operatorname{randn}(1, n), \\
& \overline{W_{9}}=0.1 \overline{W_{8}}+0.2 \operatorname{randn}(1, n), \\
& \overline{W_{10}}=0.5 \operatorname{randn}(1, n) .
\end{aligned}
$$

From 130th to 1000th sampling point, the influence of fault patterns $D_{1}$ becomes greater

$$
f_{1}=g_{1}\left(1-e^{-t_{1} / \tau_{1}}\right),
$$

where $g_{1}=6, t_{1} \in[0,13], \tau_{1}=8$.

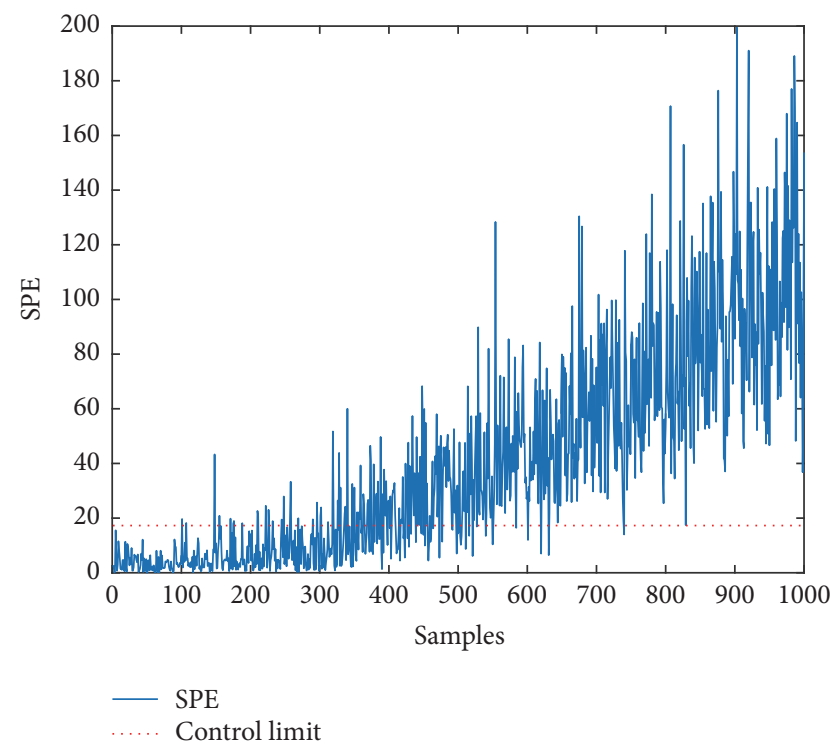

Figure 1: PCA based fault detection.

From 300th to 1000th sampling point, the influence of fault patterns $D_{7}$ becomes greater

$$
f_{7}=g_{7}\left(1-e^{-t_{7} / \tau_{7}}\right),
$$

where $g_{7}=4, t_{7} \in[1,8], \tau_{7}=6$.

4.2. Data Feature Extraction Based on DCA. Figure 1 shows SPE chart of PCA. The control limit with confidence level $\alpha=0.003$, which can be calculated by (4). From Figure 1, the abnormally can be detected from the 411th sample time. It can be concluded from Figure 1 that the detection time using PCA is not consistent with the failure point of any component.

Therefore, DCA is introduced to overcome the pattern compounding deficiency of PCA. DCA-based faults diagnosis results are depicted in Figure 2. The dotted red line is the control limit. As shown in Figure 2, the Shewhart charts correspond to $D_{1}, D_{7}$ beyond the upper control limit from 451 and 501, respectively. The results indicate that DCA is an effective multiple faults diagnosis method for multiple faults diagnosis.

4.3. Early Fault Feature Extraction Based on DCA. In this part, wavelet based denoising technique is used to extract early fault feature. The filtered SPE chart of PCA is shown in Figure 3. The blue line denotes the filtered SPE (abbreviated as F-SPE in the following); the black point line denotes the filtered SPE after smoothing (abbreviated as F-S-SPE in the following). The fault trend can be detected from the 154th sample point.

Figure 4 shows the faults results of DCA preprocessed by wavelet filter (WF-DCA). The blue line denotes the filtered designated components (the F-dcs); the black line denotes the filtered dcs after smoothing (the F-S-dcs). It can be seen from Figure 4 that the fault trend of the 1 st $\mathrm{dc}$ can be detected at 161. The 7 th $\mathrm{dc}$ is abnormal from 326. 

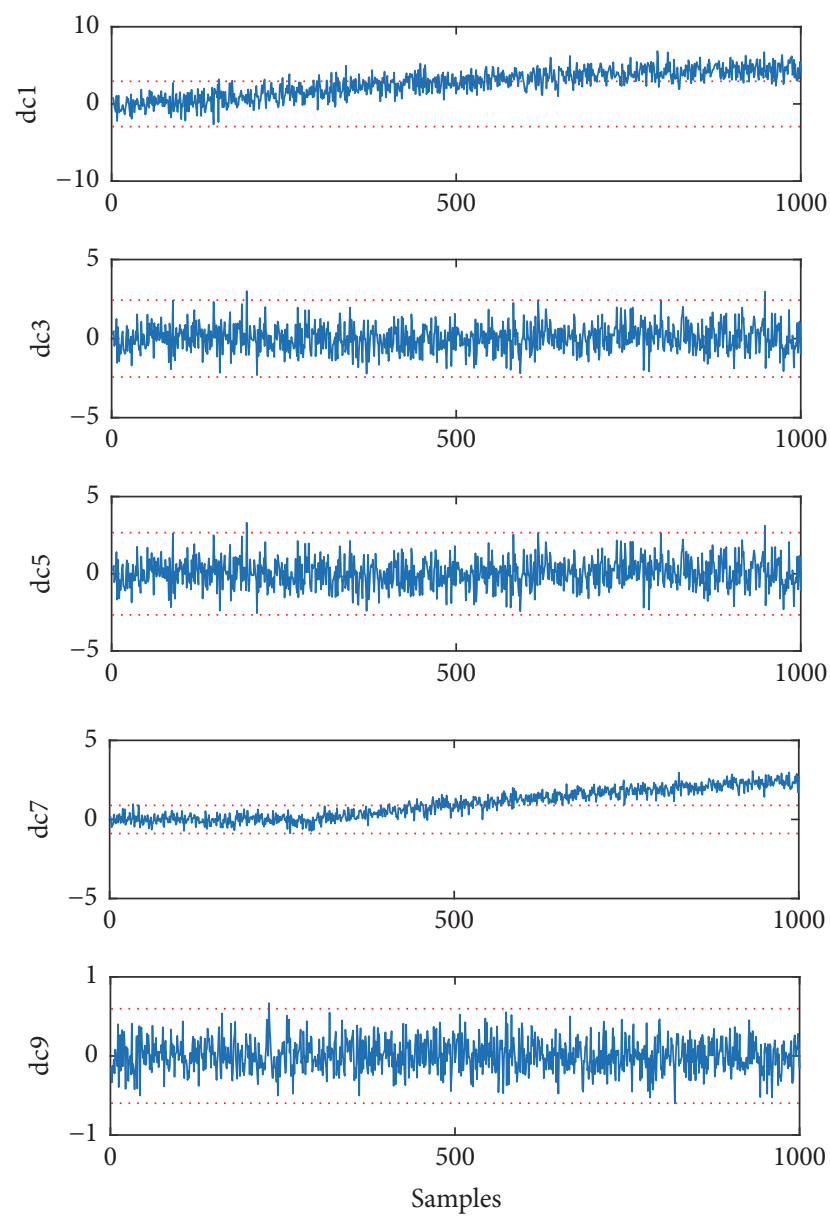
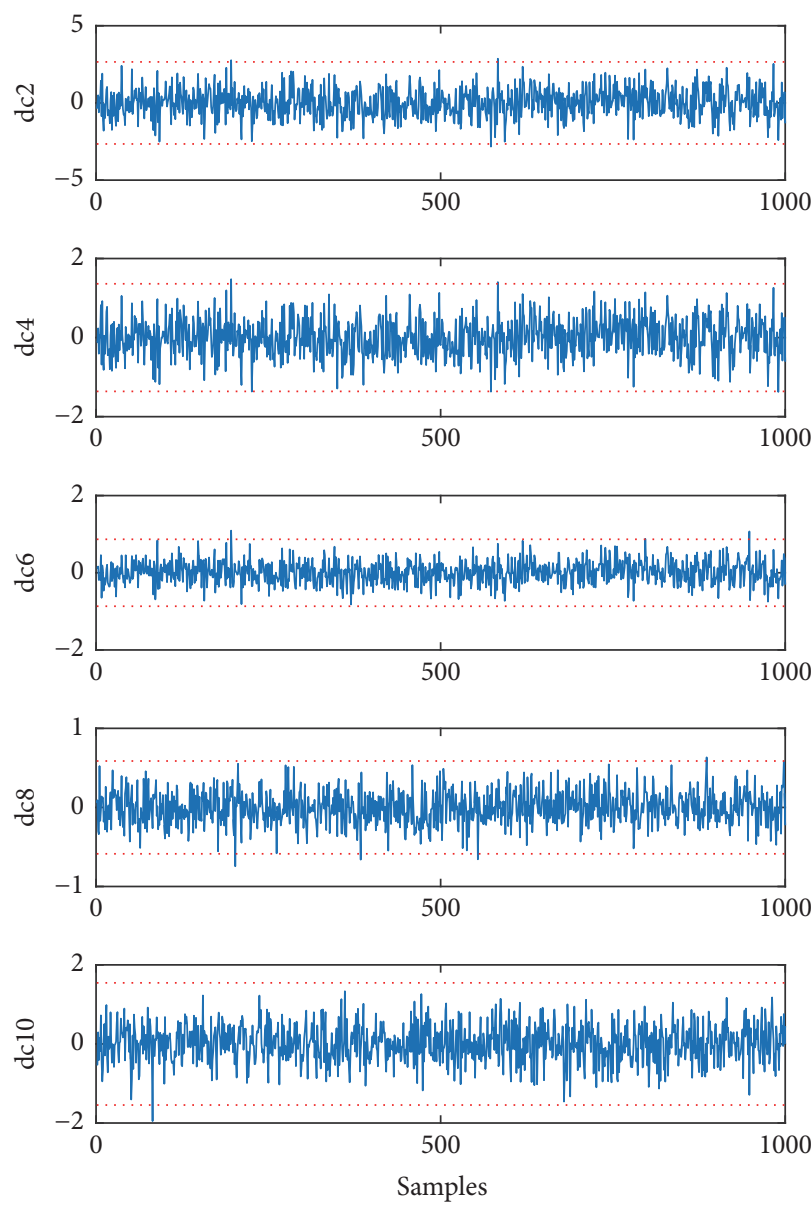

FIGURE 2: DCA-based faults diagnosis.

These simulation results imply that the early fault detection can be achieved by wavelet filtering which can increase the signal-to-noise ratio (SNR) of the fault signal. This paper determines the fault trend detection point using wavelet filtering technique.

4.4. RUL Prediction Model. In this paper, the system component corresponding to $D_{1}$ is assumed to be a critical component. In order to get more precise RUL prediction model, smoothing technique is used to postprocess the fault precursor. The smoothed evolution precursor between fault trend detection point and failure point can be defined as the damage precursor to establish RUL prediction model.

For establishing RUL prediction model based on WFPCA, the failure time $t_{f}=411$ can be determined from Figure 1. The fault trend detection point $t_{s}=154 \mathrm{can}$ be obtained from Figure 3. The F-S-SPE between 154 and 411 can be defined as the fault damage precursor. Use RUL $=a \times e^{b R}$ as the fitting function to establish RUL prediction model, where $R$ is the deviation between the value of F-S-SPE and the control limit. Figure 5 shows the fitted RUL prediction model based on WF-PCA.

Figure 6 shows the fitted RUL prediction model of the critical component based on WF-DCA. The first F-S-dc between 161 and 451 can be defined as fault damage precursor,

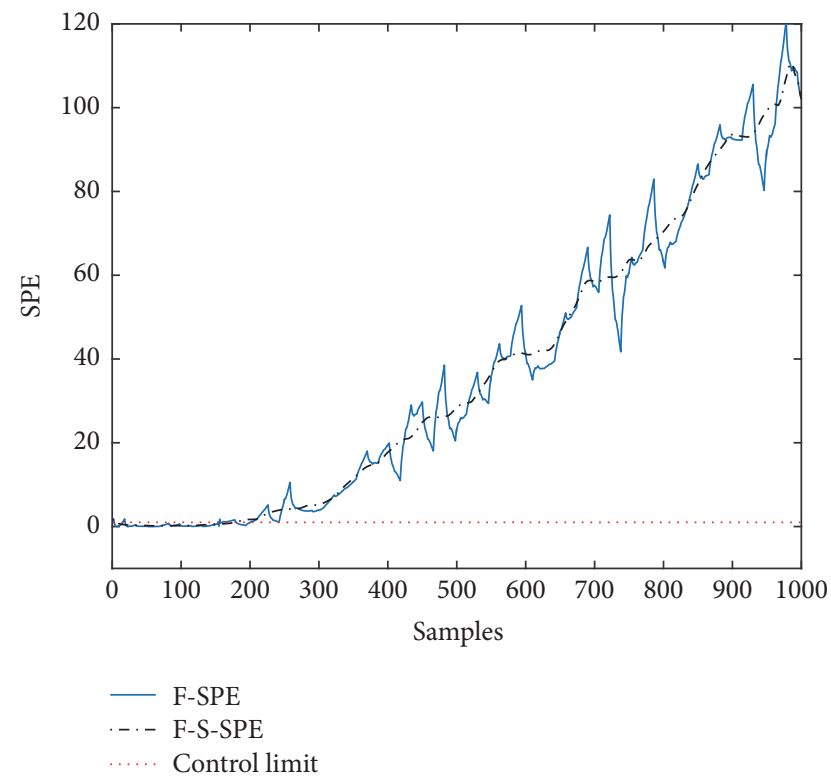

FIGURE 3: WF-PCA based early fault detection.

where $t_{f}$ and $t_{s}$ can be obtained from Figures 2 and 4, respectively. 

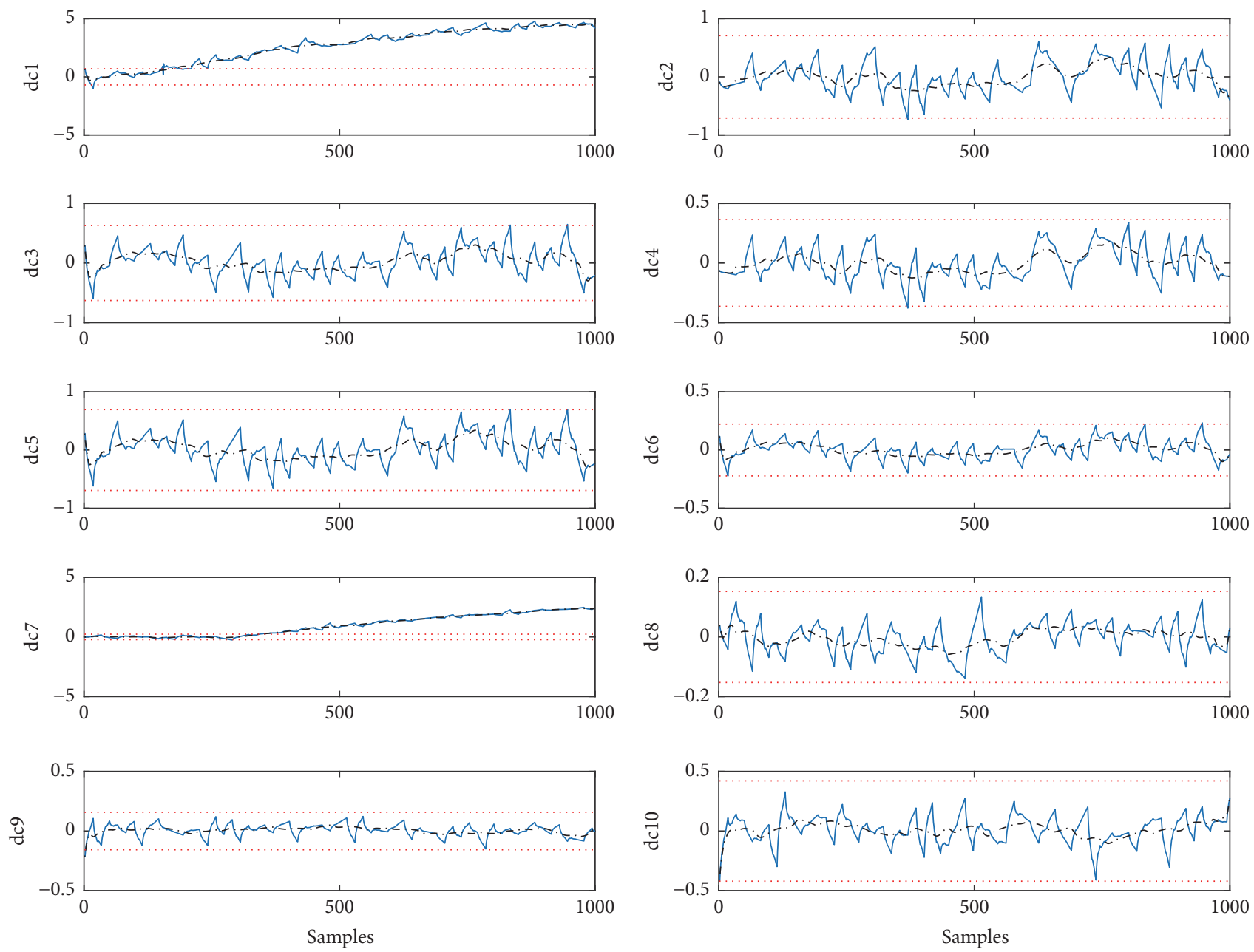

FIGURE 4: WF-DCA-based early faults diagnosis.

4.5. Online Life Prediction and Fault Diagnosis Based on DCA. Once online data is obtained, we can predict the online RUL based on the aforementioned prediction model. Figure 7 depicts the online fault detection results by online F-S-SPE. It can be seen from Figure 7 that the fault trend can be detected from the 152nd sample point.

To further demonstrate the superiority of the proposed method, two commonly used prediction algorithms are employed for comparison, namely, AR and BPNN. For BPNN and $\mathrm{AR}$, fault trend prediction and recursive RUL prediction are implemented in two separate stages which makes them unavailable to predicting the RUL online. The parameters of BPNN are shown in Table 2. In this paper, the order of AR model is 1 , and the regression parameter is $\varphi$. The recursive RUL prediction process can be formulated as follows:

$$
\begin{aligned}
\overline{W_{1 z}}(k+s)=\varphi \overline{W_{1 z}}(k+s-1) \geq q, & \\
& k=t_{s}, t_{s}+1, \ldots, t_{f},
\end{aligned}
$$

where $s$ is the number of recursive steps and $q$ is the failure control limit.

Figure 8 shows the online RUL prediction result of PCA. The blue line is the real RUL. The dotted red line is the
TABLE 2: Parameters of BPNN in pretraining.

\begin{tabular}{lc}
\hline Parameters & BPNN \\
\hline Layers & 3 \\
Neurons in each layer & {$[1,10,1]$} \\
Learning rate & 0.0001 \\
Epochs & 1000 \\
\hline
\end{tabular}

predicted RUL by the method proposed in this paper. The dotted black line is the predicted RUL by AR. The dotted blue line is the predicted RUL by BPNN. As we can see from Figure 8, the prediction accuracy of the dotted red line is higher than that of the dotted black line and the dotted blue line. Extra recursive steps are required for both AR and BPNN based RUL prediction model. The method proposed in this paper can avoid this problem and reduce the prediction error.

Figure 9 depicts the online faults diagnosis results based on WF-DCA. From Figure 9, the fault trends corresponding to $D_{1}$ and $D_{7}$ can be detected from 165 and 324, respectively. Figure 10 shows the online prediction result of the critical component. The blue line is the real RUL. The dotted red 


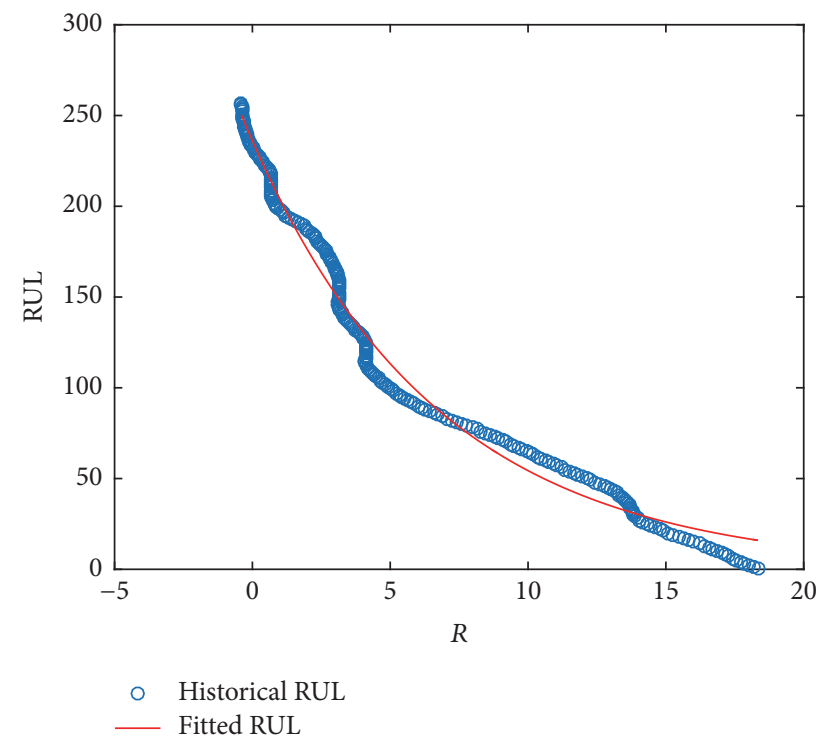

FIgURE 5: The fitted RUL prediction model based on PCA.

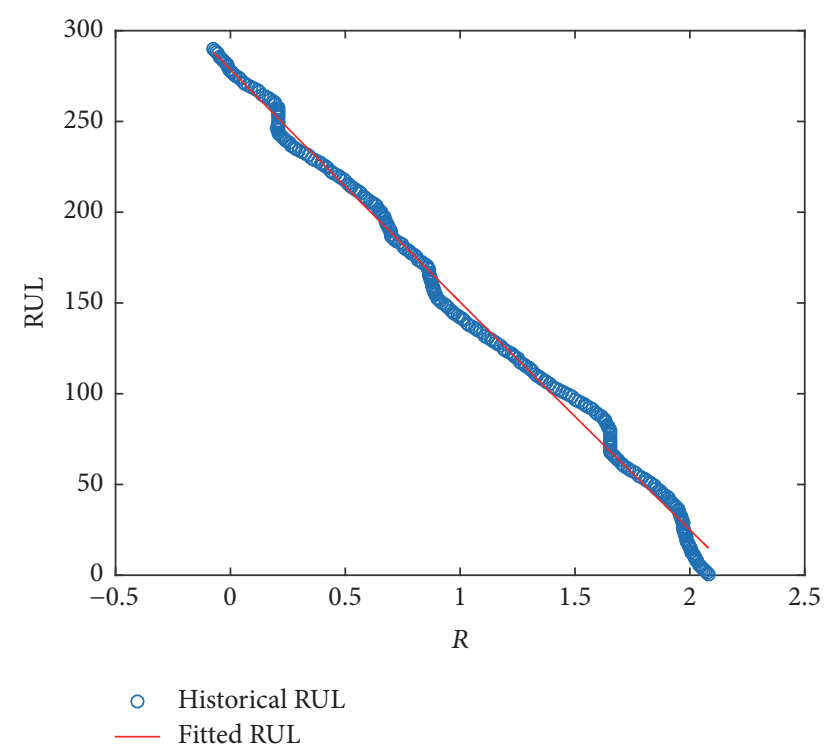

FIGURE 6: The fitted RUL prediction model of the critical component based on DCA.

TABLE 3: Mean of RUL prediction error.

\begin{tabular}{lccc}
\hline Statistics & Fitting & AR & BPNN \\
\hline SPE & 10.536 & 15.969 & 14.450 \\
dcl & 9.973 & 24.156 & 20.035 \\
\hline
\end{tabular}

line is the predicted RUL by nonlinear fitting method. The dotted black line is the predicted RUL by AR. The dotted blue line is the predicted RUL by BPNN. It can be seen from Figure 10 that fitted RUL prediction model is more efficient than other models. The mean of RUL prediction error is listed in Table 3. It can be concluded from Table 3 that the proposed method is a good choice for the RUL prediction of the critical component.

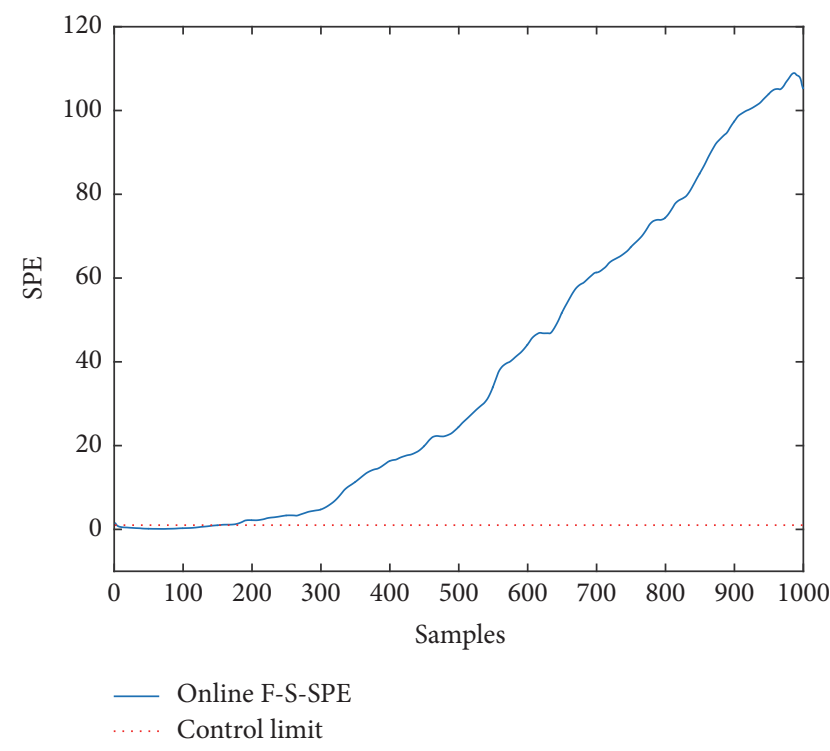

FIGURE 7: Online early fault detection based on WF-PCA.

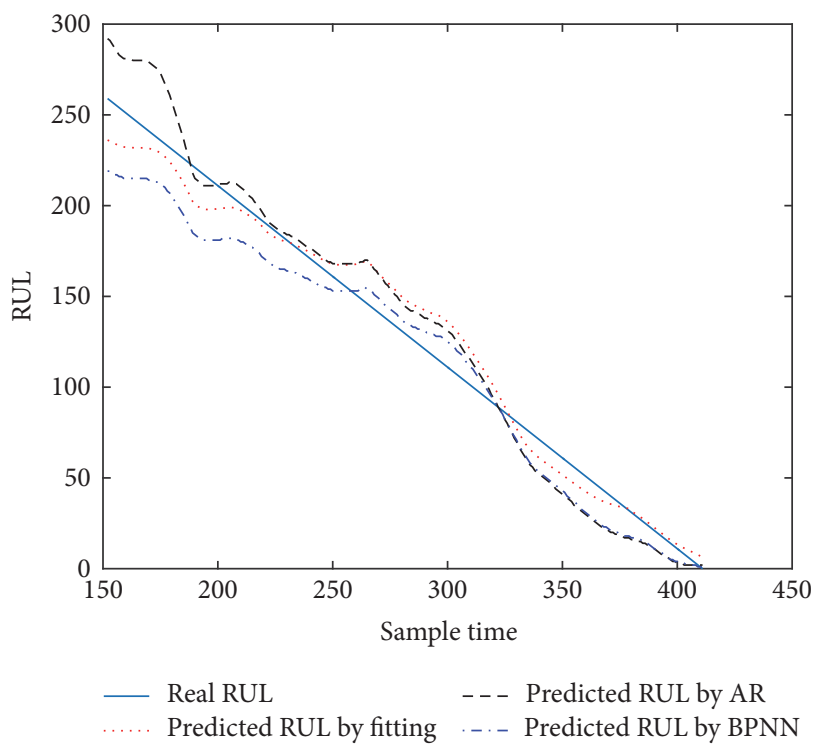

FIgURE 8: Online RUL prediction based on PCA.

\section{Conclusions}

Traditional RUL prediction is based on autoregression which cannot ensure a real-time RUL prediction since necessary computation time is required for online recursive prediction. In addition in order to implement RUL prediction for critical component that has disastrous impact on the system, DCA is introduced as a fault feature extraction tool for a certain system component to overcome the pattern compounding problem of PCA. The fault feature extracted by DCA can be used to define the fault precursor. For the sake of establishing RUL prediction model, wavelet filter technology based preprocessing is used for early detection of those slowly varying faults. Finally, exponential fitting based online RUL 

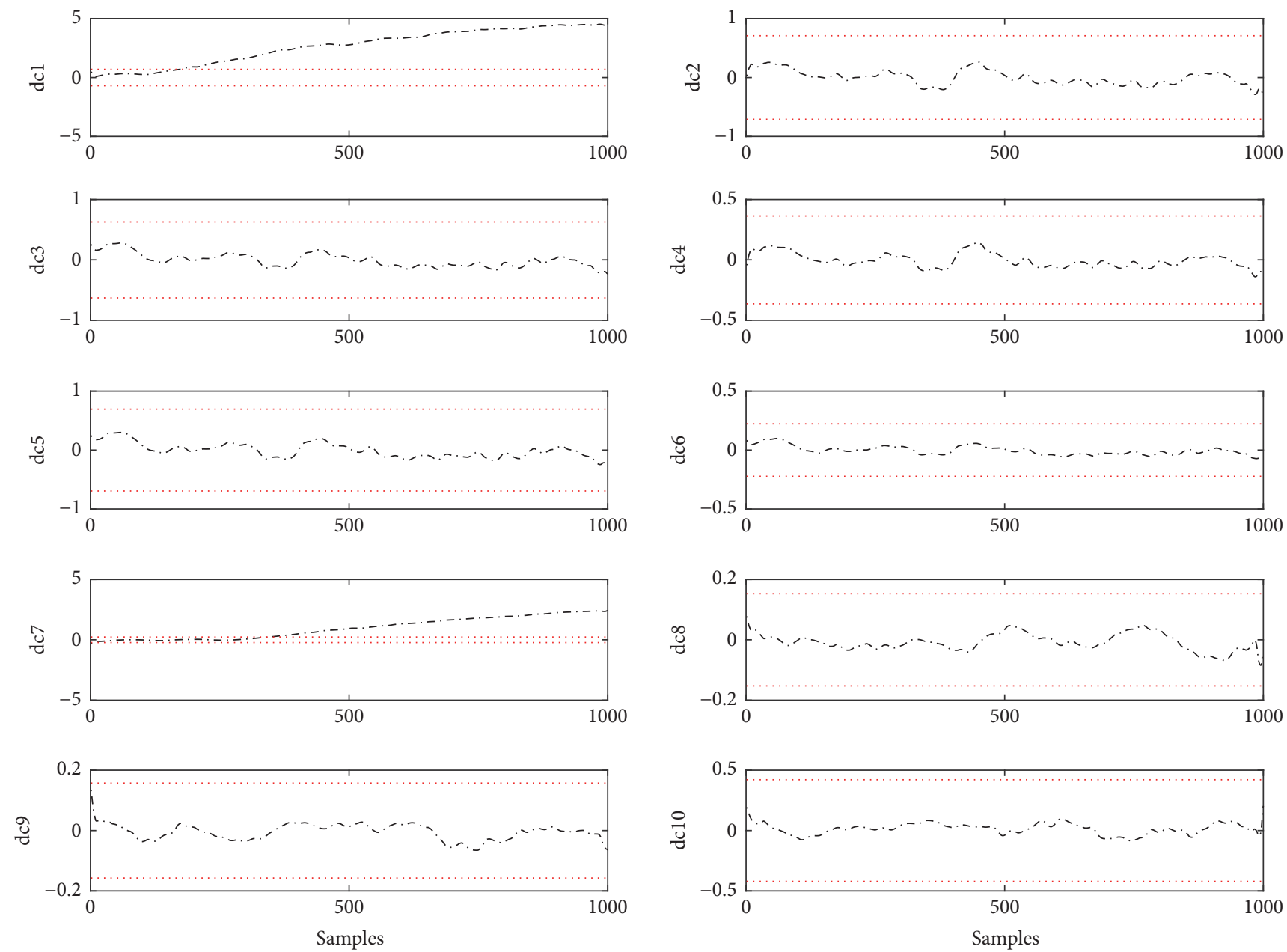

FIGURE 9: Online early fault diagnosis based on DCA.

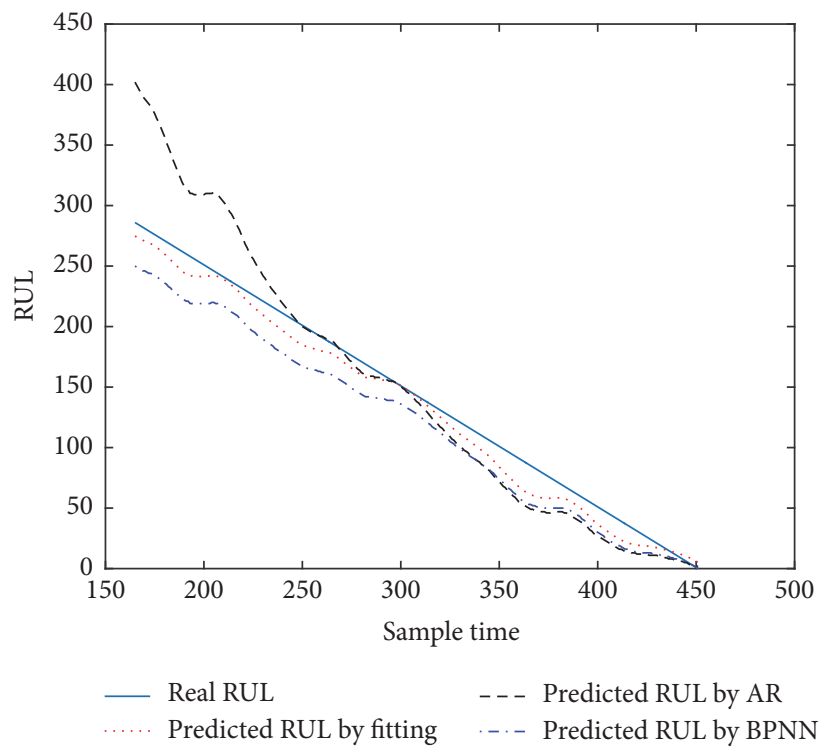

FIGURE 10: The online prediction result of the critical component. 
prediction model is developed to get the real-time online prediction of RUL.

\section{Conflicts of Interest}

The authors declare that they have no conflicts of interest.

\section{Acknowledgments}

This research was supported in part by the Natural Science Fund of China (Grant no. U1604158) and Technical Innovation Talents Scheme of Henan Province (Grant no. 2012HASTIT005).

\section{References}

[1] Y.-T. Hu, C.-H. Hu, X.-Y. Kong, and Z.-J. Zhou, "Real-time lifetime prediction method based on wavelet support vector regression and fuzzy c-means clustering," Acta Automatica Sinica, vol. 38, no. 3, pp. 331-340, 2012 (Chinese).

[2] C. Jin-Yin, H. Hui-Hao, Z. Xiao, and Y. Dong-Yong, "Fault diagnosis research for power system based on timing constraint fuzzy directed graph," International Journal of Control and Automation, vol. 9, no. 1, pp. 323-346, 2016.

[3] J. Uddin, M. R. Islam, J.-M. Kim, and C.-H. Kim, "A two-dimensional fault diagnosis model of induction motors using a gabor filter on segmented images," International Journal of Control and Automation, vol. 9, no. 1, pp. 11-22, 2016.

[4] J. Lou, K. Shan, and J. Xu, "A new condition monitoring method for wind turbines based on power curve model," International Journal of Control and Automation, vol. 121, pp. 470-475, 2016.

[5] K. X. Peng, L. MA, and K. Zhang, "Review of quality-related fault detection and diagnosis techniques for complex industrial processes," Acta Automatica Sinica, vol. 43, no. 3, pp. 349-365, 2017 (Chinese).

[6] Y. Chen, "Reference-related component analysis: a new method inheriting the advantages of PLS and PCA for separating interesting information and reducing data dimension," Chemometrics and Intelligent Laboratory Systems, vol. 156, pp. 196-202, 2016.

[7] Z. He, H. Zhou, J. Wang, and S. Zhai, "A unified framework for contrast research of the latent variable multivariate regression methods," Chemometrics and Intelligent Laboratory Systems, vol. 143, pp. 136-145, 2015.

[8] C. Zhao and F. Gao, "Online fault prognosis with relative deviation analysis and vector autoregressive modeling," Chemical Engineering Science, vol. 138, pp. 531-543, 2015.

[9] Z. Xu, Y. Ji, and D. Zhou, "A new real-time reliability prediction method for dynamic systems based on on-line fault prediction," IEEE Transactions on Reliability, vol. 58, no. 3, pp. 523-538, 2009.

[10] Z. H. Cheng, Z. Y. Yang, J. M. Zhao, Y. B. Wang, and Z. W. $\mathrm{Li}$, "Preventive maintenance strategy optimizing model under two-dimensional warranty policy," Eksploatacja i Niezawodnosc - Maintenance and Reliability, vol. 17, no. 3, pp. 365-373, 2015.

[11] L. Kumar, S. Misra, and S. K. Rath, "An empirical analysis of the effectiveness of software metrics and fault prediction model for identifying faulty classes," Computer Standards Interfaces, vol. 53, pp. 1-32, 2017.

[12] Z. Sheng, J. Wang, Z. Liu, and L. Dong, "The monitoring of CNC machine processing condition based on the BP neural network," Advanced Materials Research, vol. 542-543, pp. 95-98, 2012.
[13] S. Aras and D. K. İpek, "A new model selection strategy in time series forecasting with artificial neural networks: IHTS," Neurocomputing, vol. 174, pp. 974-987, 2016.

[14] Z. Tang and P. A. Fishwick, "Feedforward neural nets as models for time series forecasting," Journal on Computing, vol. 5, no. 4, pp. 374-385, 1993.

[15] X. Y. Wen, P. C. Li, J. N. Chen, Y. Zhu, and X. F. Wu, “Optimized BP neural network algorithm in predicting camellia oleifera yield," Journal of Northeast Forestry University, vol. 10, pp. 5660, 2016.

[16] P. Poonnoy, A. Tansakul, and M. Chinnan, "Artificial neural network modeling for temperature and moisture content prediction in tomato slices undergoing microwave-vacuum drying," Journal of Food Science, vol. 72, no. 1, pp. E42-E47, 2007.

[17] D. Liu, Z. Yu, Z. Hao, C. Zhu, and Q. Ju, "On the development of improved artificial neural network model and its application on hydrological forecasting," in Proceeding of the 3rd International Conference on Natural Computation, ICNC 2007, pp. 45-49, August 2007.

[18] W.-F. Yang and F.-L. Zeng, "Parameter estimation of the AR model based on interval analysis," in Proceeding of the 2nd International Symposium on Intelligent Information Technology Application Workshop, IITA 2008, pp. 990-993, December 2008.

[19] T. Xu, J. Wu, Z.-S. Wu, and Q. Li, "Long-term sunspot number prediction based on EMD analysis and AR model," Chinese Journal of Astronomy and Astrophysics, vol. 8, no. 3, pp. 337-342, 2008 (Chinese).

[20] X. K. Wei, Y. H. Li, P. Zhang, and J. M. Lu, "Analysis and applications of time series forecasting model via support vector machines," in Systems Engineering and Electronics, vol. 03, pp. 529-532, 2005.

[21] Y. Y. Ni and J. X. Zhang, "Modeling and forecasting for multivariate time series using a vector autoregression model," Chinese Journal of Health Statistics, vol. 31, no. 1, pp. 53-56, 2014 (Chinese).

[22] P. Do Van, E. Levrat, A. Voisin, and B. Iung, "Remaining useful life (RUL) based maintenance decision making for deteriorating systems," in Proceedings of the IFAC Workshop on Advanced Maintenance Engineering, Services and Technology, (A-MEST '12), pp. 66-72, November 2012.

[23] G. Li and D. Zhou, "SPM-based online fault prediction approach for multivariate continuous processes," Journal of Chemical Industry and Engineering, vol. 59, no. 7, pp. 1829-1833, 2008 (Chinese).

[24] G. Li, S. J. Qin, Y. Ji, and D. Zhou, "Reconstruction based fault prognosis for continuous processes," Control Engineering Practice, vol. 18, no. 10, pp. 1211-1219, 2010.

[25] R. Lewis and G. C. Reinsel, "Prediction of multivariate time series by autoregressive model fitting," Journal of Multivariate Analysis, vol. 16, no. 3, pp. 393-411, 1985.

[26] X.-S. Si, W. Wang, C.-H. Hu, and D.-H. Zhou, "Remaining useful life estimation - a review on the statistical data driven approaches," European Journal of Operational Research, vol. 213, no. 1, pp. 1-14, 2011.

[27] J. Ma, Q. Y. Wang, and G. Li, "Fault prediction of purbine machine based PCA fault reconstruction method," Automation and Information Engineering, vol. 03, pp. 25-28, 2011.

[28] F. N. Zhou, C. L. Wen, Z. G. Chen, and Y. B. Leng, "DCA based multi-level small fault diagnosis method," Chinese Journal of Electronics, vol. 38, no. 8, pp. 1874-1879, 2010 (Chinese). 
[29] F. N. Zhou, Multi-Fault Diagnosis Method and Application Based on Statistical Feature Extraction [Ph.D. dissertation], Shanghai Maritime University, Shanghai, China, 2009.

[30] M. Panda and P. Khilar, "Distributed self fault diagnosis algorithm for large scale wireless sensor networks using modified three sigma edit test," Ad Hoc Networks, vol. 25, pp. 170-184, 2015.

[31] T. A. Brunner, "Relative merits of using maximum error versus 3(sigma) in describing the performance of laser-exposure reticle writing systems," in The International Society for Optical Engineering, vol. 2440 of Proceedings of SPIE, pp. 550-559, 1995.

[32] R. Ganesan, "Real-time monitoring of complex sensor data using wavelet-based multiresolution analysis," International Journal of Advanced Manufacturing Technology, vol. 39, no. 56, pp. 543-558, 2008.

[33] H. Li, X. Yang, J. Li, J. Chen, and C. Cheng, “The maximum energy of wavelet decomposition approximation -related adaptive wavelet de-nosing for partial discharge UHF pulse in GIS," Transactions of China Electrotechnical Society, vol. 27, no. 5, pp. 84-91, 2012. 


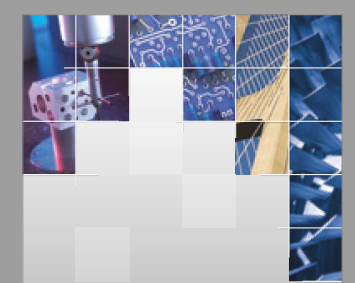

\section{Enfincering}
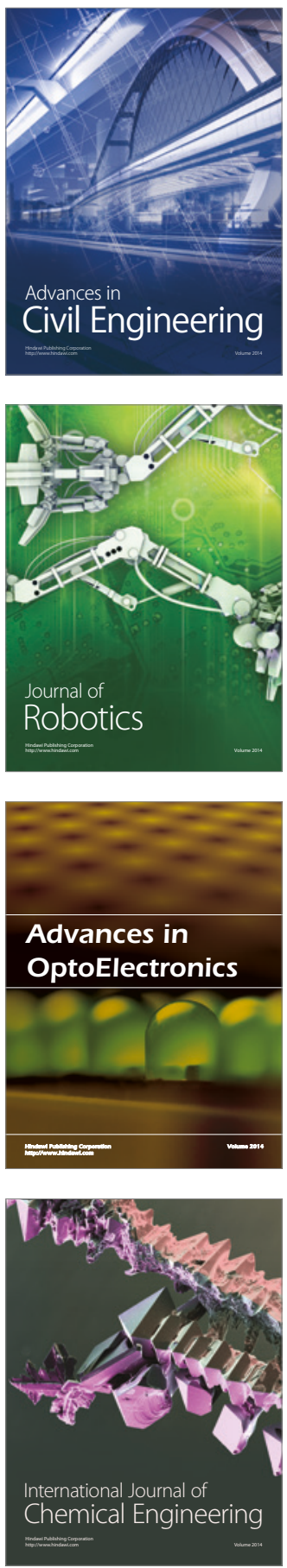

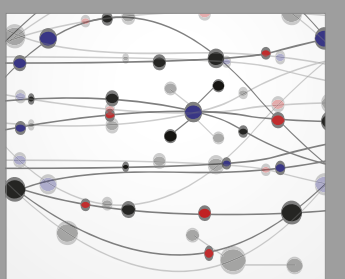

The Scientific World Journal

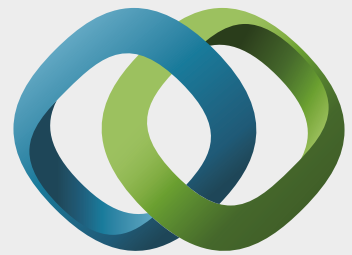

\section{Hindawi}

Submit your manuscripts at

https://www.hindawi.com
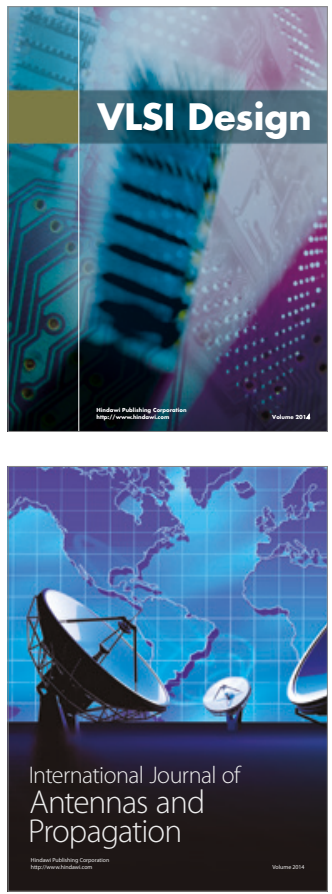

\section{Rotating}

Machinery
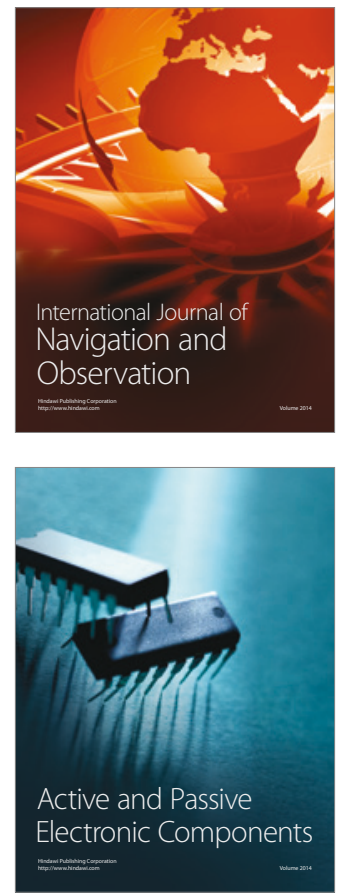
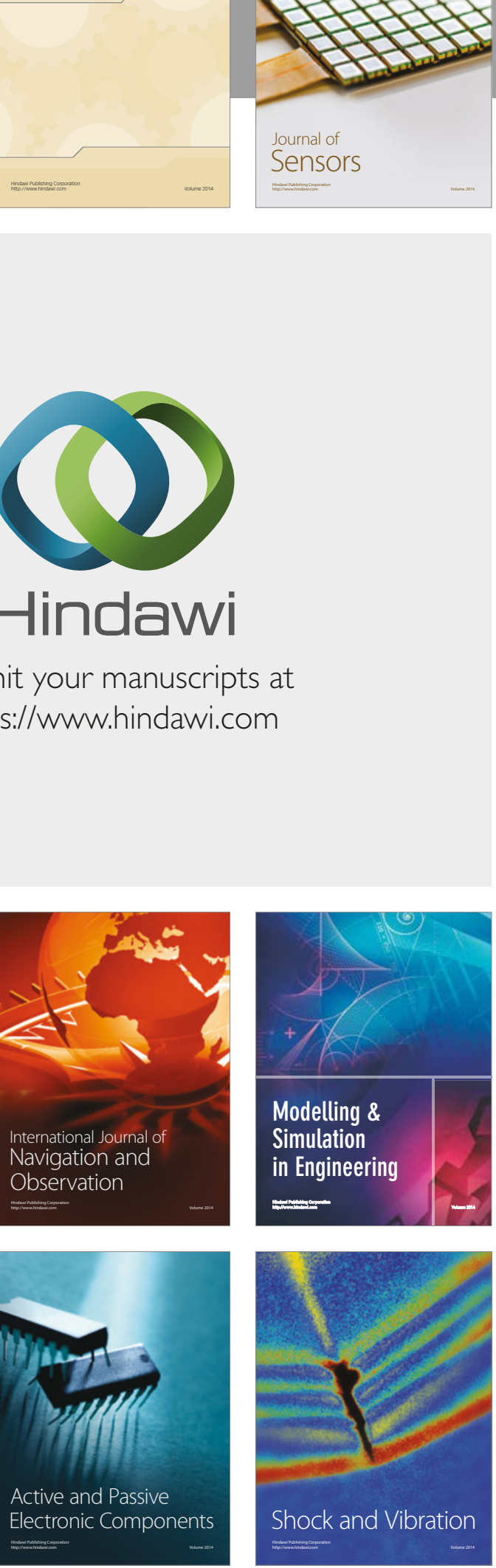
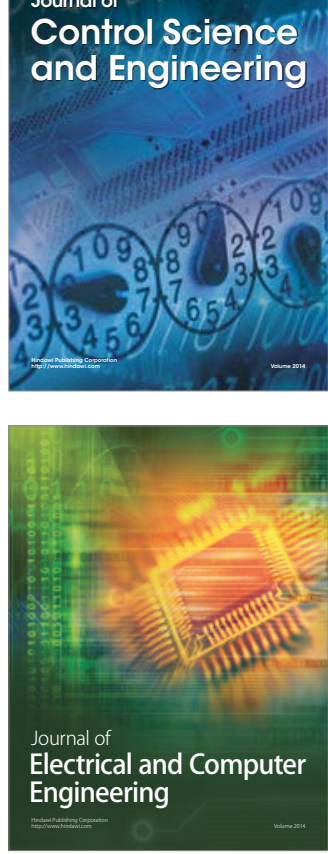

Distributed

Journal of

Control Science

and Engineering
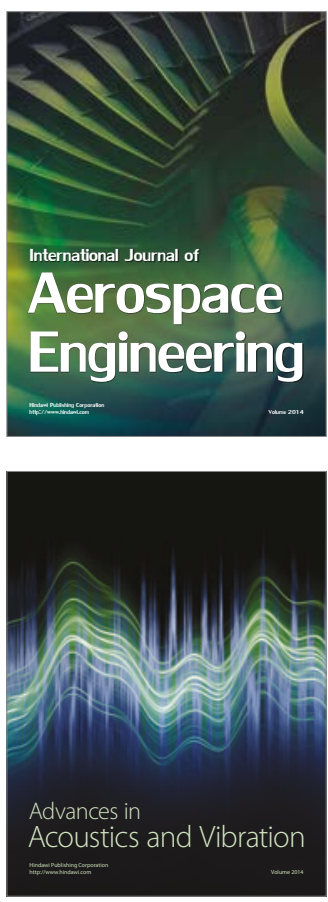

Sensor Networks 